\title{
Recent results of decay spectroscopy at RIBF
}

\author{
Hiroshi Watanabe $1,2,3$,a \\ and RIBF Decay Spectroscopy Collaboration in 2009, EURICA U-Beam Collaboration in 2012 \\ ${ }^{1}$ RIKEN Nishina Center, 2-1 Hirosawa, Wako, Saitama 351-0198, Japan \\ ${ }^{2}$ International Research Center for Nuclei and Particles in the Cosmos, Beihang University, Beijing 100191, China \\ ${ }^{3}$ School of Physics and Nuclear Energy Engineering, Beihang University, Beijing 100191, China
}

\begin{abstract}
The neutron-rich nuclei ${ }^{109} \mathrm{Nb}$ and ${ }^{110} \mathrm{Mo}$ have been investigated by means of $\beta-\gamma$ and isomer spectroscopy following in-flight fission of a ${ }^{238} \mathrm{U}$ beam at $345 \mathrm{MeV} /$ nucleon at the RIBF facility. A $T_{1 / 2}=150$ (30) ns isomer at $313 \mathrm{keV}$ has been identified in ${ }^{109} \mathrm{Nb}$. The low-lying levels in ${ }^{109} \mathrm{Nb}$ have been also populated following the $\beta$-decay of ${ }^{109} \mathrm{Zr}$. The observed hindrances of the electromagnetic transitions deexciting the isomeric state are discussed in terms of possible shape coexistence. For ${ }^{110} \mathrm{Mo}$, spectroscopic information on the low-lying levels of the quasi- $\gamma$ band built on the $2_{2}^{+}$state at $494 \mathrm{keV}$ has been obtained for the first time. The experimental finding of the $2_{2}^{+}$state being lower than the $4_{1}^{+}$level suggests that axially-asymmetric $\gamma$ softness is substantially enhanced in this nucleus. The experimental results are compared with model calculations based on the general Bohr Hamiltonian method. A new project of decay spectroscopy at RIBF, named EURICA, is also introduced.
\end{abstract}

\section{Introduction}

Gamma-ray spectroscopy following the $\beta$ decay is an effective tool for exploring low-lying yrast and non-yrast states, which provide key structure information such as the shape transitions/coexistence and the single-particle levels. For the study of rare isotopes, especially when the nucleus of interest lies at the boundaries of availability for spectroscopic studies, isomeric decays are likely to be a more useful means than $\beta$ decays to populate lowerlying levels. The identification of such characteristic isomers will pin down currently controversial subjects including the evolution of shell structures. The combined $\beta$ - and isomeric-decay measurements at the RI Beam Factory (RIBF) [1], which has the capability of providing the world's strongest RI beams, are at the forefront of exploration of exotic nuclei far from the stability line.

This paper focuses on the achievements obtained in the first decay spectroscopy at RIBF in 2009. A major aim of this work was the study of neutron-rich nuclei around $Z=40$ and $A=110$, where the shape transitions from prolate, via $\gamma$-soft, to oblate deformations are predicted to occur with increasing the number of neutrons. New results obtained include the observation of shape evolution in ${ }^{106,108} \mathrm{Zr}$ [2], a possible oblate-shape isomer in ${ }^{109} \mathrm{Nb}$ [3], and a large-amplitude $\gamma$-soft dynamics in ${ }^{110} \mathrm{Mo}$ [4]. These findings revealed the shape-transitional phenomena in this neutron-rich region for the first time.

Research opportunities for decay spectroscopy at RIBF will be expanded with the high-efficient $\gamma$-ray

\footnotetext{
ae-mail: hiroshi@ribf.riken.jp
}

spectrometer EURICA (EUROBALL-RIKEN Cluster Array) [5]. The present status of this project will be also introduced.

\section{Decay spectroscopy experiment in 2009}

\subsection{Experimental details}

Experiments were carried out at the RIBF facility, cooperated by RIKEN Nishina Center and CNS, University of Tokyo. Neutron-rich $A \approx 110$ nuclei were produced via inflight fission of ${ }^{238} \mathrm{U}^{86+}$ projectiles at $345 \mathrm{MeV} /$ nucleon, incident on a beryllium target with a thickness of $3 \mathrm{~mm}$. The average beam intensity was approximately $0.3 \mathrm{pnA}$ during the experiment. The nuclei of interest were separated and transported through the BigRIPS spectrometer [6] [7], operated with a 6-mm-thick wedge-shaped aluminum degrader at the first dispersive focal plane for purification of the secondary beams. An additional degrader placed at the second dispersive focus served as a charge stripper to remove fragments that were not fully stripped. The identification of nuclei by their atomic number and the mass-to-charge ratio was achieved on the basis of the $\Delta E$ TOF- $B \rho$ method, where $\Delta E$, TOF, and $B \rho$ denote energy loss, time of flight, and magnetic rigidity, respectively. The identified nuclei were implanted into an active stopper consisting of nine double-sided silicon-strip detectors (DSSSD) stacked compactly. Each DSSSD has a thickness of $1 \mathrm{~mm}$ with a $50 \mathrm{~mm} \times 50 \mathrm{~mm}$ active area segmented into sixteen strips on both sides in the vertical and horizontal dimensions. The DSSSDs also served as detectors for 
electrons following $\beta$-decay and internal-conversion processes. The implantation of an identified particle was associated with the subsequent electron events that were detected in the same DSSSD pixel. Gamma rays were detected by four Compton-suppressed Clover-type Ge detectors arranged around the DSSSD telescope in a close geometry. Further details of a particle-identification spectrum and data-analysis techniques are given in Ref. [3].

\subsection{Results and discussion}

\subsection{1 ${ }^{109} \mathrm{Nb}$}

Prior to the present work, nothing has been reported on excited states in ${ }^{109} \mathrm{Nb}$. As shown in Fig. 1(a), the $\gamma$ rays at energies of 117, 196, and $313 \mathrm{keV}$ have been unambiguously identified in delayed coincidence with ${ }^{109} \mathrm{Nb}$ ions. Figure 1(c) exhibits the sum of three time spectra relative to the implantation of ${ }^{109} \mathrm{Nb}$, from which a half-life of 150(30) ns was deduced. As can be seen in Fig. 1(b), the $117-\mathrm{keV}$ line was clearly identified in a $\beta$-gated $\gamma$-ray spectrum following the decay of ${ }^{109} \mathrm{Zr}$, while the other two transitions might be populated very weakly. Based on the difference in feeding pattern between the isomeric and $\beta$ decays and the consistency in energy with the level at 313 $\mathrm{keV}$, we propose the decay scheme from the $T_{1 / 2}=150(30)$ ns isomeric state in ${ }^{109} \mathrm{Nb}$ shown in Fig. 1(d).

The isomeric state is identified at $313 \mathrm{keV}$, which is much less than the pairing gaps for both protons and neutrons in this region, indicating that this isomer should have a single-proton configuration. With deduced $\gamma$-ray intensities for the two branches from the $T_{1 / 2}=150(30) \mathrm{ns}$ isomer, the transition strengths for possible multipolarities are evaluated in Table 1. This procedure virtually rules out $M 2$ and higher-multipole possibilities for both the 313- and 196-keV transitions because their strengths exceed a recommended upper limit for each multipolarity. Although $E 1$ transitions could occur with large hindrances relative to the Weisskopf single-particle estimate, the $B(E 1)$ values for the $\gamma$ rays deexciting the isomer in ${ }^{109} \mathrm{Nb}$ would be two or three orders of magnitude smaller than the $E 1$ strengths observed for ${ }^{103,103} \mathrm{Nb}$ [8] [9]. In a similar way, the isomeric-decay transitions would be extremely retarded given an $M 1$ multipolarity, that might be unlikely to take place as the main decay branch if the isomer were similar in structure to the low-lying states. Assuming that the isomeric decay transitions are of E2 character, the implied transition probabilities are 0.024 and 0.15 W.u. for the 313- and 196-keV $\gamma$ rays, respectively.

A possible explanation for the occurrence of electromagnetic transitions with strong hindrances can be given in terms of shape difference between the isomeric state and the the states to which the isomer decays. The configuration-constrained method [10] was employed to calculate potential energy surfaces (PES) of the singleproton configurations and to compare the excitation energies. The PES calculation suggests that the lowest-lying oblate-deformed state has the $7 / 2[413], K^{\pi}=7 / 2^{+}$configuration [3]. Note that the calculation predicts the prolate $3 / 2[301], K^{\pi}=3 / 2^{-}$configuration to be the lowest

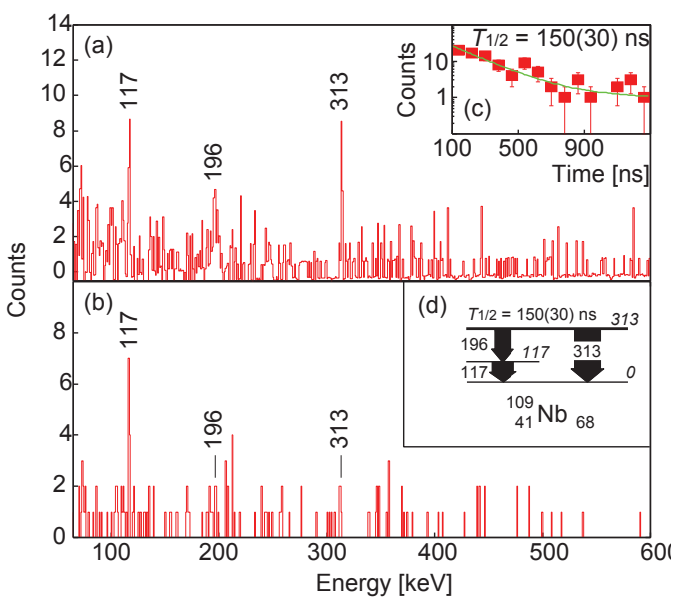

Figure 1. $\gamma$-ray spectra measured (a) with a particle gate on ${ }^{109} \mathrm{Nb}$ within $150-750 \mathrm{~ns}$ and (b) in coincidence with $\beta$ rays detected within $0-170 \mathrm{~ms}$ after implantation of ${ }^{109} \mathrm{Zr}$. The inset (c) shows the time distribution and associated fit for $\gamma$-ray coincidence events relative to the beam implantation with a sum of gates on the 117-, 196-, and 313-keV transitions in ${ }^{109} \mathrm{Nb}$ in the nanosecond range. Panel (d) shows a partial level scheme of ${ }^{109} \mathrm{Nb}$ established in the present work.

state, while the ground state is expected to have spin and parity of $5 / 2^{+}$from the systematics for the $\mathrm{Nb}$ isotopes with odd mass [11]. Based on the argument on the transition strengths as already discussed, a $7 / 2^{+} \rightarrow 3 / 2^{-}, M 2$ transition is unlikely. Assuming the spin and parity of the isomeric state to be $7 / 2^{+}$, the ground state is more likely to have $5 / 2^{+}$or $5 / 2^{-}$. Irrespective of the spin and parity of the low-lying states, the electromagnetic transitions that deexcite the oblate-deformed state should be strongly inhibited due to the substantial shape change involved in its decay.

\subsection{2 ${ }^{110} \mathrm{Mo}$}

The left panel in Fig. 2 exhibits the level scheme of ${ }^{110} \mathrm{Mo}$. Prior to the present work, the ground-state band in ${ }^{110} \mathrm{Mo}$ has been known up to the $10^{+}$state by measuring the prompt $\gamma$ rays from the spontaneous fission of ${ }^{248} \mathrm{Cm}$ [12]. In addition to the 214-, 386-, and 532-keV $\gamma$ rays that belong to the ground-state band, seven new transitions have been unambiguously observed in a singles $\gamma$-ray spectrum measured in coincidence with $\beta$ rays subsequent to implantation of ${ }^{110} \mathrm{Nb}$, as shown in the right panel in Fig. 2.

The second $2^{+}$level $\left(2_{2}^{+}\right)$is proposed at $494 \mathrm{keV}$, decaying by the 281 - and $494-\mathrm{keV}$ transitions which directly feed the yrast $2^{+}$and $0^{+}$states, respectively. The assignment of the $2_{2}^{+}$state at $494 \mathrm{keV}$ is justified, since only the energy sum for the 281-214-keV cascade agrees with the energy of the 494-keV $\gamma$ ray within experimental errors among the newly observed $\gamma$ rays. The $207-\mathrm{keV}$ transition is assigned as feeding the $2_{2}^{+}$state on account of the consistency in energy with the level at $701 \mathrm{keV}$, which is most likely the $3^{+}$member of the quasi- $\gamma$ band decaying also via the parallel deexcitation pathway that consists of the 214- 
Table 1. $\gamma$-ray relative intensities and $B(\sigma \lambda)$ values for transitions depopulating the $T_{1 / 2}=150(30)$ ns isomeric state in ${ }^{109} \mathrm{Nb}$, assuming different multipolarities.

\begin{tabular}{cccccc}
\hline \hline $\begin{array}{c}E_{\gamma} \\
(\mathrm{keV})\end{array}$ & $\begin{array}{c}I_{\gamma} \\
(\text { relative) }\end{array}$ & $E 1$ & $\begin{array}{c}B(\sigma \lambda) \text { (W.u.) } \\
M 1\end{array}$ & $E 2$ & $M 2$ \\
\hline 313.1 & $62(20)$ & $3.9(13) \times 10^{-8}$ & $2.9(10) \times 10^{-6}$ & $2.4(8) \times 10^{-2}$ & $1.8(6)$ \\
196.3 & $38(14)$ & $1.0(4) \times 10^{-7}$ & $7.2(26) \times 10^{-6}$ & $1.5(5) \times 10^{-1}$ & $1.1(4) \times 10^{1}$ \\
\hline \hline
\end{tabular}
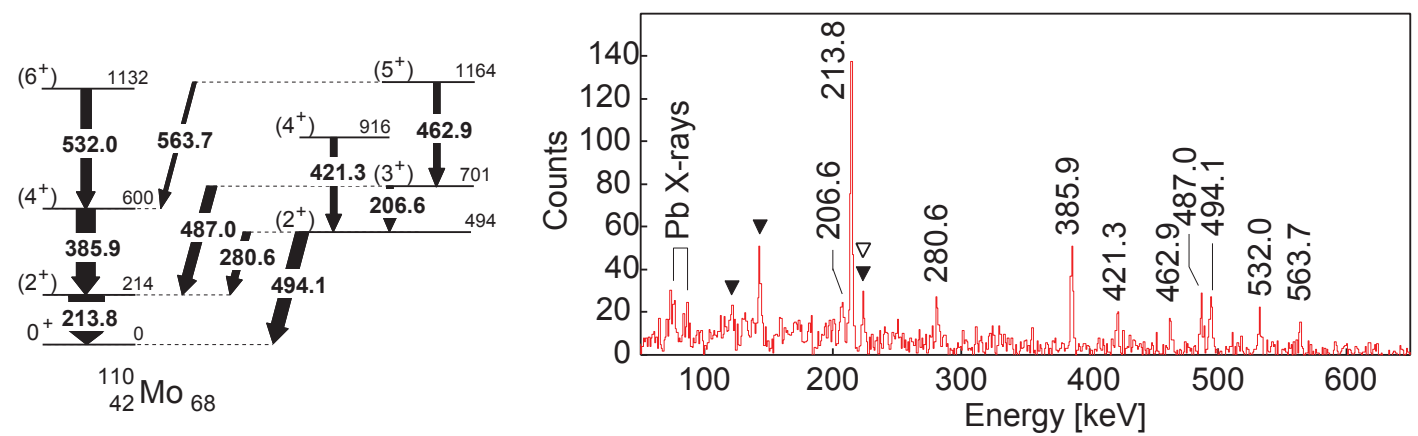

Figure 2. Partial level scheme of ${ }^{110} \mathrm{Mo}$ (left) and $\gamma$-ray spectrum measured in coincidence with $\beta$ rays detected within $250 \mathrm{~ms}$ after implantation of ${ }^{110} \mathrm{Nb}$ (right). Contaminants from ${ }^{110} \mathrm{Tc}$ and ${ }^{109} \mathrm{Mo}$ are indicated with filled and open triangles, respectively.

and $487-\mathrm{keV}$ transitions. The $\gamma$ rays at 421 and $463 \mathrm{keV}$ are proposed to be the $4_{2}^{+} \rightarrow 2_{2}^{+}$and $5_{1}^{+} \rightarrow 3_{1}^{+}$transitions, respectively, based on the systematics of the quasi- $\gamma$-band levels for lighter Mo isotopes. Furthermore, the measured intensity of the 532-keV $\gamma$ ray depopulating the yrast $6^{+}$ state at $1132 \mathrm{keV}$ implies a sizable value of spin $(\approx 5 \hbar)$ for the $\beta$-decaying state in ${ }^{110} \mathrm{Nb}$, consistent with the argument above on the population of the $4_{2}^{+}$and $5_{1}^{+}$levels at 916 and $1164 \mathrm{keV}$, respectively, in the $\beta$ decay of ${ }^{110} \mathrm{Nb}$.

The experimental excitation energies of the $2_{1}^{+}, 4_{1}^{+}$, and $2_{2}^{+}$states in even Mo isotopes are reproduced well in a microscopic theory based on the general Bohr Hamiltonian approach [13], in which the potential energy and inertial functions (mass parameters) are calculated using the constrained Hartree-Fock-Bogoliubov (CHFB) method with the Skyrme effective interaction. For ${ }^{110} \mathrm{Mo}$, the $2_{2}^{+}$state is predicted to lie at about $500 \mathrm{keV}$ (see Fig. 6 in Ref. [13]), being in good agreement with the energy assigned in the present work. The level energies of the ground-state and quasi- $\gamma$ bands calculated using the SIII and SLy4 versions of the Skyrme interaction are shown in Figs. 3(A) and 3(B), respectively. We also performed a similar calculation with the pairing-plus-quadrupole $(\mathrm{P}+\mathrm{Q})$ model including a quadrupole pairing [14], in which the inertial functions are calculated with the local quasiparticle random-phase approximation (LQRPA). The result of this calculation is shown in Fig. 3(C).

Figure 3 provides the comparison of the experimental levels with the predictions of the two theoretical frameworks. The agreement between the observed and calculated level energies is very satisfactory for both the ground-state and quasi- $\gamma$ bands. In particular, it is noteworthy that the observation for the $2_{2}^{+}$state being lower than the $4_{1}^{+}$level is reproduced in all of the calculations.
In the right panels in Fig. 3, the potential energy surface calculations for ${ }^{110}$ Mo exhibit local minima at the prolate $\left(\gamma \approx 0^{\circ}\right)$ and oblate $\left(\gamma \approx 60^{\circ}\right)$ sides using the SIII and SLy4 versions of the Skyrme interaction, respectively. However, the exact location of the energy minimum is not important in characterizing the collective level properties of the heavier Mo isotopes, because an overall profile in the potential energy surface spreads over the $\gamma$ degree of freedom. Consequently, the level structure of ${ }^{110} \mathrm{Mo}$ is ascribed to its $\gamma$-soft nature rather than the rigid deformation of any kind. More discussion on the structural evolution in even-even $A \approx 110$ nuclei is developed in Ref. [4] in comparison with the level properties in the neutron-rich $A \approx 190$ region.

\section{The EURICA project}

EURICA is an acronym for the EUROBALL-RIKEN Cluster Array, consisting of twelve Cluster-type detectors, each of which contains seven HPGe crystals packed closely and housed on a common cryostat, which were developed for the former EUROBALL $\gamma$-ray spectrometer [15]. This is an ambitious project of decay spectroscopy for exotic nuclei which lie far from the $\beta$-stability line, in conjunction with highly intense RI beams at the RIBF facility. The clusters are mounted on the support frame that was employed in the RISING stopped-beam experiments [16] and located at the final focus of the BigRIPS-ZeroDegree spectrometer. The signals from each individual pre-amplifier are sent to two branches, one using the Digital Gamma Finder (DGF) modules that can provide both energy and timing information based on time stamps with $25 \mathrm{~ns}$ resolution, and the other using analogue 

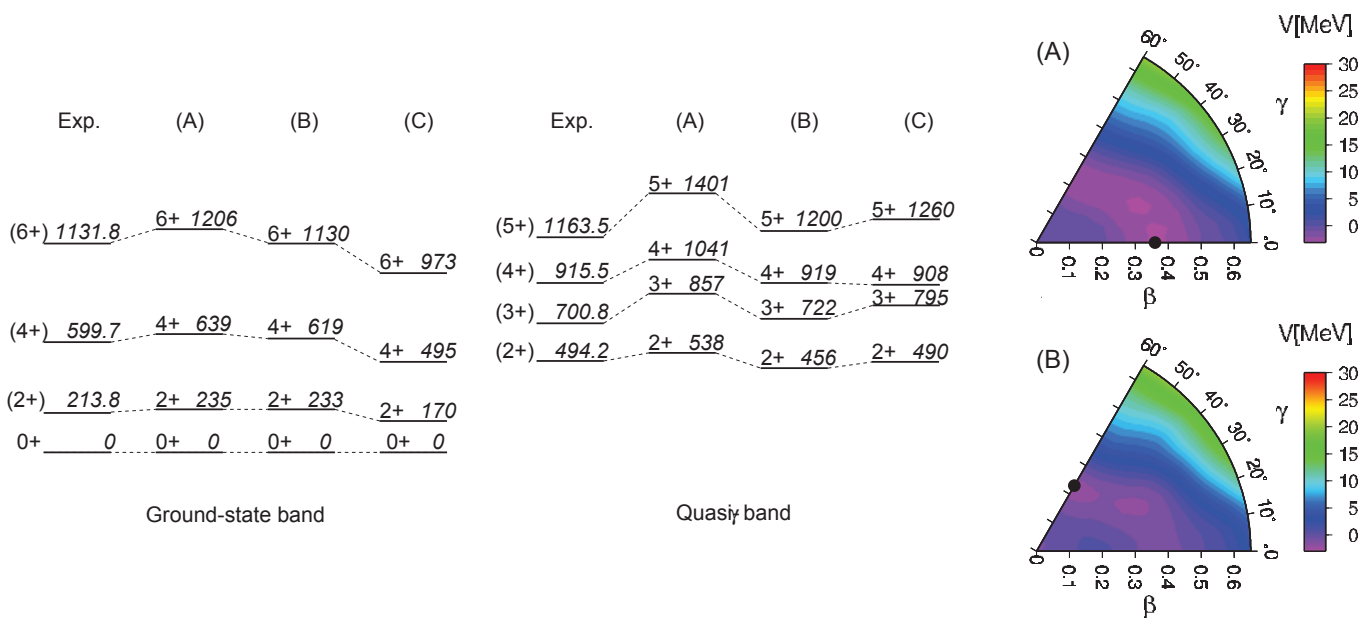

Figure 3. Experimental and calculated level energies (in keV) for the ground-state (left) and quasi- $\gamma$ bands (middle) in ${ }^{110} \mathrm{Mo}$. The model calculations are based on the general Bohr Hamiltonian approach with the CHFB+IB method using the SIII (A) and SLy4 (B) versions of the Skyrme interaction, and the CHFB+LQRPA method using the P+Q force $(\mathrm{C})$. Right panels show potential energy surface calculations for ${ }^{110}$ Mo with the SIII (A) and SLy4 (B) versions of the Skyrme interaction. The energy minima are indicated with filled circles.

timing electronics being composed of conventional TFACFD-TDC modules.

The EURICA spectrometer is coupled with a highlysegmented active stopper, named WAS3ABi (wide-range active silicon-strip stopper array for beta and ion detection) [17], which consists of eight double-sided siliconstrip detectors (DSSSD) stacked compactly. Each DSSSD has a thickness of $1 \mathrm{~mm}$ with an active area segmented into sixty and forty strips (1-mm pitch) on each side in the horizontal and vertical dimensions, respectively. This composite detector system is a powerful tool to resolve weak $\gamma$ rays following the $\beta$ decay of a specific isotope under the conditions of cocktail beams.

To realize the measurement of level half-lives ranging from a few hundred picoseconds to a few nanoseconds, we install $\mathrm{LaBr}(\mathrm{Ce})_{3}$ scintillation detectors from the UK fast-timing project into the EURICA setup. Eighteen $\phi 1.5^{\prime \prime} \times 2^{\prime \prime}$-length detectors are arranged in three vacant slots with six crystals each in the EURICA support frame. Time resolution is typically $280 \mathrm{ps}$ full width at half maximum between pairs of detectors for $\gamma$ rays at $500 \mathrm{keV}$. In addition, BC-418 plastic scintillators with a thickness of $2 \mathrm{~mm}$ are arranged both upstream and downstream of WAS3ABi in a close geometry so as to cover the active area of DSSSD. $\beta$ rays which eject out of WAS3ABi can be detected by these plastic counters, giving rise to the time zero for coincident $\gamma$ rays detected by the $\mathrm{LaBr}_{3}(\mathrm{Ce})$ detectors, and the difference in time between the plastic and $\mathrm{LaBr}_{3}(\mathrm{Ce})$ signals provides information on the halflife for the intermediate level.
The first long-term EURICA experimental campaign has been carried out in November and December in 2012. Very neutron-rich nuclei in the vicinity of the doublymagic nuclei ${ }^{78} \mathrm{Ni}$ and ${ }^{132} \mathrm{Sn}$ were produced by in-flight fission of ${ }^{238} \mathrm{U}$ beams with an intensity of 7-12 pnA. The detailed analysis of $\beta$-decay half-lives and isomer and $\beta$ delayed $\gamma$-ray spectroscopy is still ongoing.

\section{References}

[1] Y. Yano, Nucl. Instrum. Methods B 261, 1009 (2007)

[2] T. Sumikama et al., Phys. Rev. Lett. 106, 202501 (2011)

[3] H. Watanabe et al., Phys. Lett. B 696, 186 (2011)

[4] H. Watanabe et al., Phys. Lett. B 704, 270 (2011)

[5] S. Nishimura, Nucl. Phys. News 22, No.3 (2012)

[6] T. Kubo, Nucl. Instrum. Methods B 204, 97 (2003)

[7] T. Ohnishi et al., J. Phys. Soc. Jpn. 77, 083201 (2008)

[8] H. Ohm et al., Z. Phys. A 340, 5 (1991)

[9] M. Liang et al., Z. Phys. A 344, 357 (1992)

[10] F. Xu et al., Phys. Lett. B 435, 257 (1998)

[11] G. Audi et al., Nucl. Phys. A 729, 3 (2003)

[12] W. Urban et al., Eur. Phys. J. A 20, 381 (2004)

[13] L. Próchniak, Int. J. Mod. Phys. E 19, 705 (2010)

[14] N. Hinohara et al., Phys. Rev. C 82, 064313 (2010)

[15] J. Simpson et al., Z. Phys. A 358, 139 (1997)

[16] S. Pietri, Nucl. Instrum. Methods B 261, 1079 (2007)

[17] S. Nishimura, Prog. Theor. Exp. Phys. 03C006 (2012) 